\title{
EDITORIAL
}

\section{The 2017 JHG Young Scientist Award}

Journal of Human Genetics (2017) 62, 1007; doi:10.1038/jhg.2017.89; published online 5 October 2017

The JHG Young Scientist Award recognizes articles that have made a

significant contribution to the Journal of Human Genetics, thanks to their scientific excellence and impact in the field of human genetics.

We are pleased to announce the winners of the 2017 JHG Young Scientist Award.

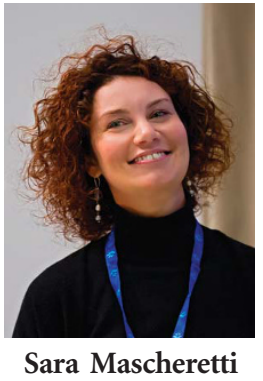

Child Psychopathology Unit, Scientific Institute, IRCCS Eugenio Medea, Lecco, Italy.

For the paper entitled 'KIAA0319 and ROBO1: evidence on association with reading and pleiotropic effects on language and mathematics abilities in developmental dyslexia', J. Hum. Genet. 59, 189-197 (2014); doi:10.1038/jhg.2013.141.

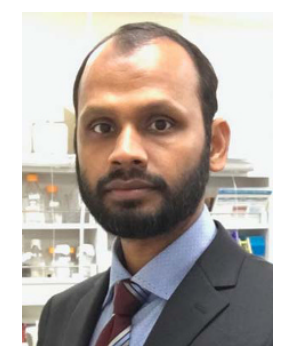

Mohammad Arif Hossain

Researcher, Advanced Clinical Research Center, Southern Tohoku Research Institute for Neuroscience, Shin Yurigaoka General Hospital, Kawasaki, Kanagawa, Japan.

For the paper entitled 'Chaperone therapy for Krabbe disease: potential for late-onset GALC mutations', J. Hum. Genet. 60, 539-545 (2015); doi:10.1038/jhg.2015.61.

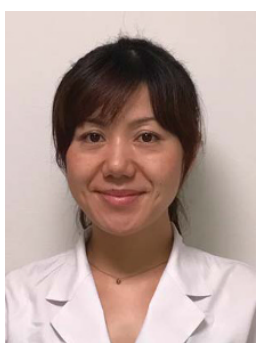

Hiroko Mataki

Department of Pulmonary Medicine, Graduate School of Medical and Dental Sciences, Kagoshima University, Kagoshima, Japan.

For the paper entitled 'Downregulation of the microRNA-1/133a cluster enhances cancer cell migration and invasion in lung-squamous cell carcinoma via regulation of Coronin1C, J. Hum. Genet. 60, 53-61 (2015); doi:10.1038/jhg.2014.111.

We would like to offer our warmest congratulations and wish them every success in their future careers.

Naomichi Matsumoto Editor-in-Chief Journal of Human Genetics E-mail:naomat@yokohama-cu.ac.jp 